\title{
Secondary Plasma Cell Leukemia in a Recurrent Multiple Myeloma: Rare Case Scenario
}

\author{
Arati A. Inamdar ${ }^{1}$, Abraham Loo ${ }^{2}$, Nagy Mikhail ${ }^{3}$, Patrick Lee ${ }^{4}$ \\ 1. Pathology, RWJBarnabas Health, Livingston, USA 2. Pathology and Laboratory Medicine, Rutgers Robert Wood \\ Johnson Medical School, New Brunswick, USA 3. Pathology, Rutgers Robert Wood Johnson Medical School, New \\ Brunswick, USA 4. Hematology Oncology, Monmouth Medical Center, Long Branch, USA
}

Corresponding author: Arati A. Inamdar, aratia.inamdar@rwjbh.org

\begin{abstract}
Plasma cell leukemia (PCL) is an aggressive hematological condition characterized by the presence of plasma cells in the peripheral smear. It presents as de novo or may arise from multiple myeloma (MM), and hence is diagnosed as primary or secondary PCL, respectively. We report a case of 79-year-old patient diagnosed with MM two years prior to the admission to our institution with prior treatment with bortezomib, lenalidomide and dexamethasone (VRD) and daratumumab, pomalidomide and dexamethasone. Morphologic examination and flow cytometry studies performed on the peripheral smear demonstrated $45 \%-55 \%$ small to medium atypical plasma cells showing a kappa restriction and dim CD138 expression on flow cytometry analysis. The patient was started on brentuximab vedotin, etoposide, cytoxan and dexamethasone, which resulted in near complete elimination of the atypical plasma cells from the peripheral smear one week after the completion of two cycles. He received three cycles of brentuximab vedotin with a gradual decrease in serum free light chain. However, he eventually developed lethargy, weakness and seizures. The involvement of the central nervous system (CNS) by MM was confirmed with MRI, flow cytometry and cytology of cerebrospinal fluid. The treatment with whole brain radiation and ibrutinib was initiated. Our case report highlights the rare case of aggressive clinical course of MM leading to the development of plasmacytoma of kidney, secondary PCL and eventually spreading to the CNS.
\end{abstract}

Received 04/27/2020

Review began 05/08/2020 Review ended 05/25/2020 Published 06/05/2020

\section{() Copyright 2020}

Inamdar et al. This is an open access article distributed under the terms of the Creative Commons Attribution License CC-BY 4.0., which permits unrestricted use, distribution, and reproduction in any medium, provided the original author and source are credited.
Categories: Pathology, Oncology, Hematology

Keywords: flow cytometry, multiple myeloma, novel immunomodulatory agents, plasmacytoma, plasma cell leukemia

\section{Introduction}

Plasma cell leukemia (PCL) is defined by the presence of $>2 \times 10^{9}$ /liter circulating plasma cells (CPCs) in the peripheral blood or by a relative plasmacytosis $>20 \%$ of blood leukocytes [1]. In rare cases $(2 \%-4 \%)$, late or advanced stage multiple myeloma (MM) may undergo clonal transformation and develop into secondary plasma cell leukemia (SPCL) [2]. Recent studies have compared the overall survival (OS) of patients with MM with percentage of CPCs in the peripheral blood. No difference in survival is noted between the patients of sPCL with 5\%-19\% and those with >20\% CPCs. Such comparative studies have advocated for a lower threshold of CPCs to define PCL [3-5]. The morphology and immunophenotype of the clonal plasma cells seen in primary PCL and SPCL are similar; hence, a clinical history of MM is crucial in establishing a diagnosis of sPCL. sPCL has a dismal prognosis with a median OS of only seven months with standard chemotherapy [6]. MM with t (11:14) is seen in $15 \%-20 \%$ of all cases and is considered as an intermediate risk with often unpredictable outcome [7]. We present a unique case of a 79-year old male with a past history of relapse/refractory MM evolving from monoclonal gammopathy of undetermined significance (MGUS) within two years followed by a relapse with plasmacytoma of the kidney. He was admitted to our institution for further management of his aggressive MM and diagnosed with PCL. Despite initial response to the treatment regimen, central nervous system (CNS) involvement by MM was revealed within four months of initial presentation. Our report highlights the rare case of aggressive form of secondary form of PCL with plasmacytoma of kidney and CNS involvement.

\section{Case Presentation}

Our case report involves a 79-year-old male with a diagnosis of MGUS at outside institution who underwent bone marrow biopsy due to persistent anemia and hypogammaglobulinemia at another institution. The biopsy specimen demonstrated normocellular marrow with $20 \%-30 \%$ cellularity along with decreased myeloid:erythrocyte (M:E) ratio due to a mild erythroid hyperplasia and mild granulocytic hypoplasia (Figure 1A). A CD138 immunohistochemical stain demonstrated a marked increase $(>10 \%)$ in plasma cells (Figure 1B). Flow cytometry studies demonstrated monoclonal kappa-positive plasma cell population, which were negative for CD56 and comprised $0.9 \%$ of total events (Figures $1 C, 1 D$ ). Fluorescence in situ hybridization (FISH) analysis demonstrated a t(11:14) (Figure 1E) without any other cytogenetic abnormalities such as $\mathrm{p} 53$, deletion of $1 \mathrm{p}(\mathrm{CDKN} 2 \mathrm{C})$, additional copy of $1 \mathrm{q}$ (CKS1B) or deletion of retinoblastoma 1. Laboratory findings showed elevated lactate dehydrogenase (LDH) with low calcium. Based on these findings, the patient was diagnosed with MM and treated with bortezomib, lenalidomide and dexamethasone (VRD). 


\section{Cureus}

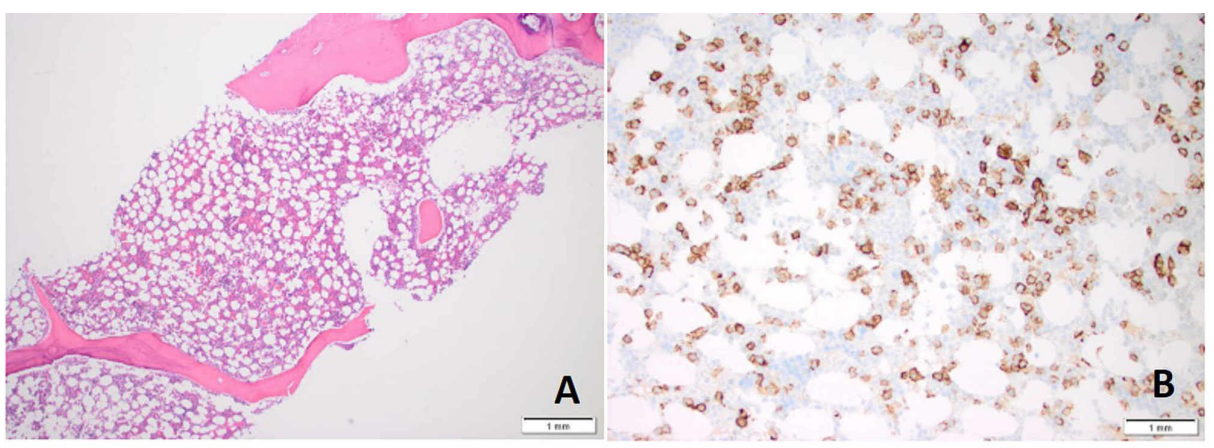

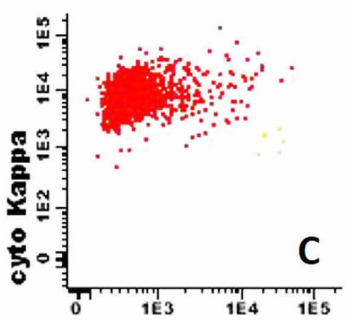

cyto Lambda
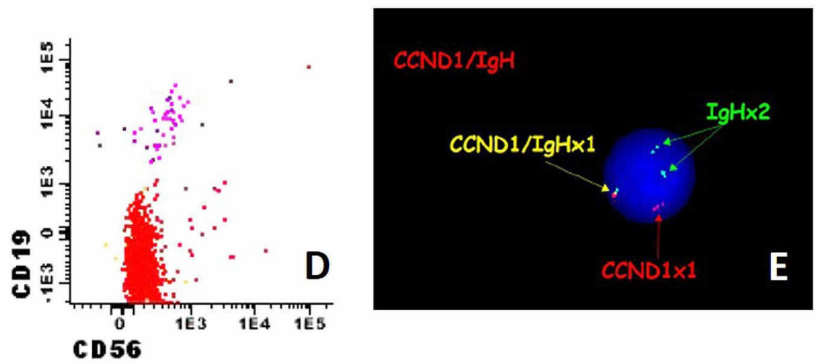

CD56

\section{FIGURE 1: Bone marrow biopsy, flow cytometry (CD38 gating) and FISH}

study for multiple myeloma.

(A) Bone marrow biopsy demonstrated normocellular marrow with $20 \%-30 \%$ cellularity along with increased CD138-positive plasma (brown colored) cells (B). (C) Bone marrow aspirate flow cytometry studies showed kappa-restricted clone, negative for CD56 (D). (E). FISH analysis using break apart probe on bone marrow aspirate detected $\mathrm{t}(11: 14)$ translocation.

FISH, Fluorescence in situ hybridization

He underwent another bone marrow biopsy almost a year later, which revealed persistent/recurrent kappa monoclonal plasma cells involving 20\%-30\% of marrow cellularity (Figure 2A). The abnormal plasma cells demonstrated weak CD138 staining by immunohistochemical stain. Kappa and lambda in situ hybridization stains demonstrated a marked kappa restriction (Figure 2B). Flow cytometry studies performed on the aspirate upon gating CD38-positive cells demonstrated $12 \%$ plasma cells with a kappa restriction and partial CD19 and CD56 expression (Figures 2C, 2D). Cytogenetics/FISH studies again showed $t(11: 14$ ) abnormality only (Figure $2 E$ ).
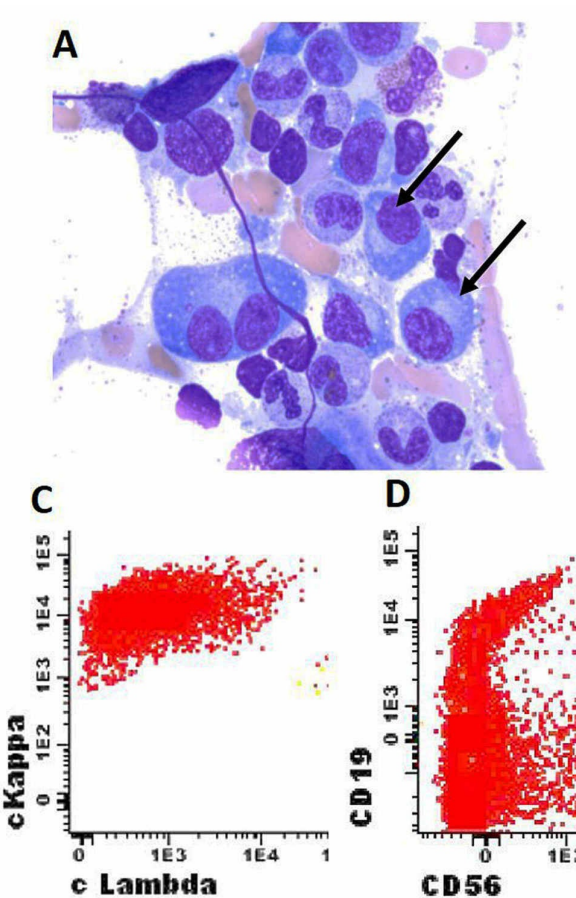

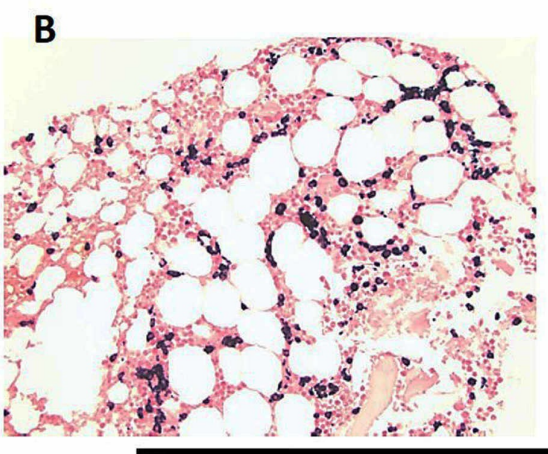

E CCND1/IgH
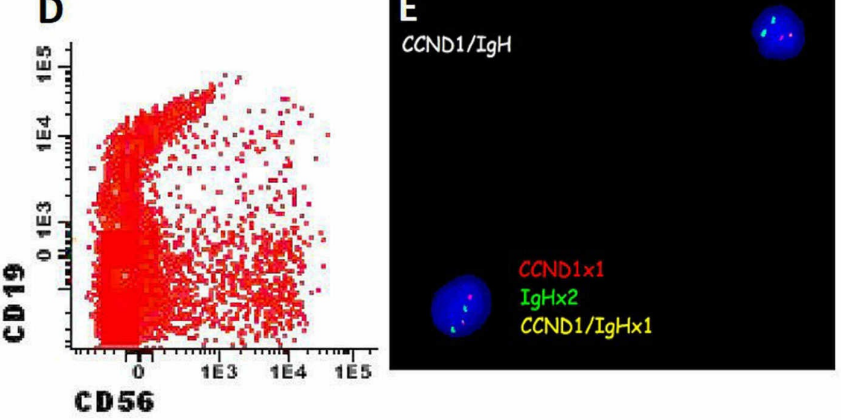


\section{Cureus}

\section{FIGURE 2: Bone marrow biopsy, flow cytometry and FISH study for recurrent multiple myeloma.}

(A) Bone marrow biopsy demonstrated plasma cells (arrows) and predominantly kappa expression by plasma (black colored) cells (B). (C). Flow cytometry studies showed a kappa-restricted clone, which was positive for CD56 (D). (E). FISH analysis using break apart probe on bone marrow aspirate detected $t(11: 14)$ translocation.

FISH, Fluorescence in situ hybridization

The patient received six cycles of daratumumab, pomalidomide and dexamethasone. The patient then developed severe abdominal pain. MRI of the abdomen and pelvis revealed a large mass in the left kidney near the hilum measuring $7.0 \times 6.0 \times 5.0 \mathrm{~cm}$ (Figure 3). A biopsy of the lesion showed diffuse sheet of atypical plasmacytoid cells with immunophenotypic profile consistent with plasmacytoma, positive for CD138, CD56, CD30 and kappa-ish, and negative for CD5, PAX5, CD20 and CD117. Flow cytometry studies demonstrated clonal plasma cells with a cytoplasmic kappa light chain (KLC) restriction and positivity for CD138, CD56, CD4 and kappa.
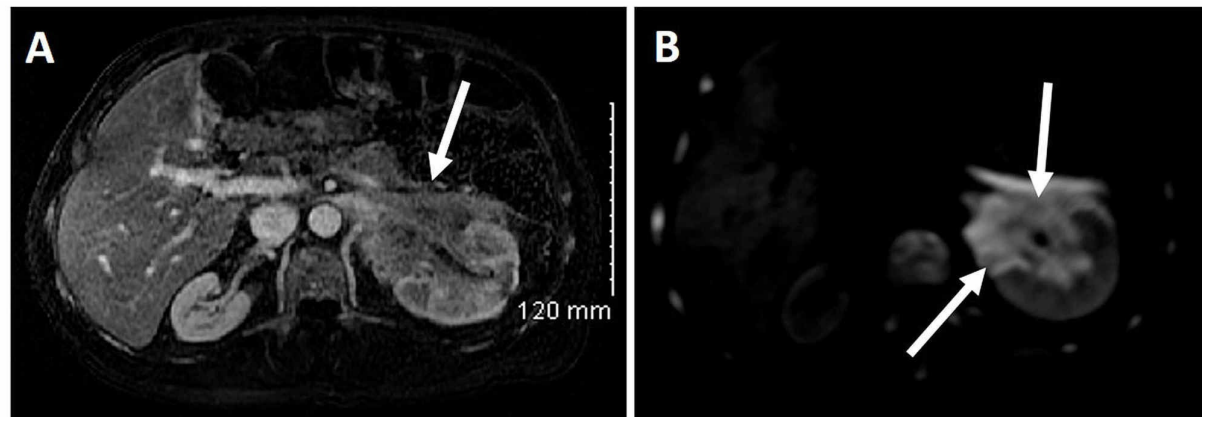

\section{FIGURE 3: MRI of abdomen and pelvis.}

(A, B) A large ill-defined mass (arrows) was identified in the left kidney near hilum as visualized via T-1 and T-2 weighted images, respectively.

With the prior history of recurrent MM not achieving remisssion and plasmacytoma of the left kidney, the patient came to our institution with progressively increasing leukocytosis, hypogammaglobulinemia and anemia. On admission, laboratory evaluation of peripheral blood showed a hemoglobin of $9.5 \mathrm{~g} / \mathrm{dL}$, a white blood cell count of $27.2 \mathrm{~K} / \mathrm{mm}^{3}$, which was interpreted by the hematology technicians as $12 \%$ atypical lymphocytes, and platelet count of $61 \mathrm{~K} / \mathrm{mm}^{3}$. Pathologist review of the peripheral smear revealed $45 \%-55 \%$ immature small to large multilobated plasma cells with fine chromatin and moderate to high nuclear:cytoplasmic ratio (Figures $4 A-4 C$ ). Flow cytometry performed on the peripheral blood confirmed PCL with 22.4\% CD138/CD38 (dim-positive) plasma cells, which were also expressing intracellular KLC (Figures $4 D-4 F)$. Interestingly, $90.5 \%$ of these plasma cells demonstrated surface kappa expression without expression of CD19 (Figures 4G,4H). Furthermore, 70\% of plasma cells showed CD56 expression (Figure 4I). Other relevant laboratory parameters include blood urea nitrogen $29 \mathrm{mg} / \mathrm{dL}$, creatinine $2.05 \mathrm{mg} / \mathrm{mL}$ and glomerular filtration rate $32 \mathrm{~mL} / \mathrm{min} / 1.73 \mathrm{~m}^{2}$. Quantitative serum protein analysis revealed low IgG, IgA and IgM of 127, 14 and $5 \mathrm{mg} / \mathrm{dL}$, respectively. The serum kappa/lambda ratio was 4.94 where kappa and lambda free were 7.9 and $1.6 \mathrm{mg} / \mathrm{L}$, respectively. The patient was started on brentuximab vedotin, etoposide, cytoxan and dexamethasone. 


\section{Cureus}
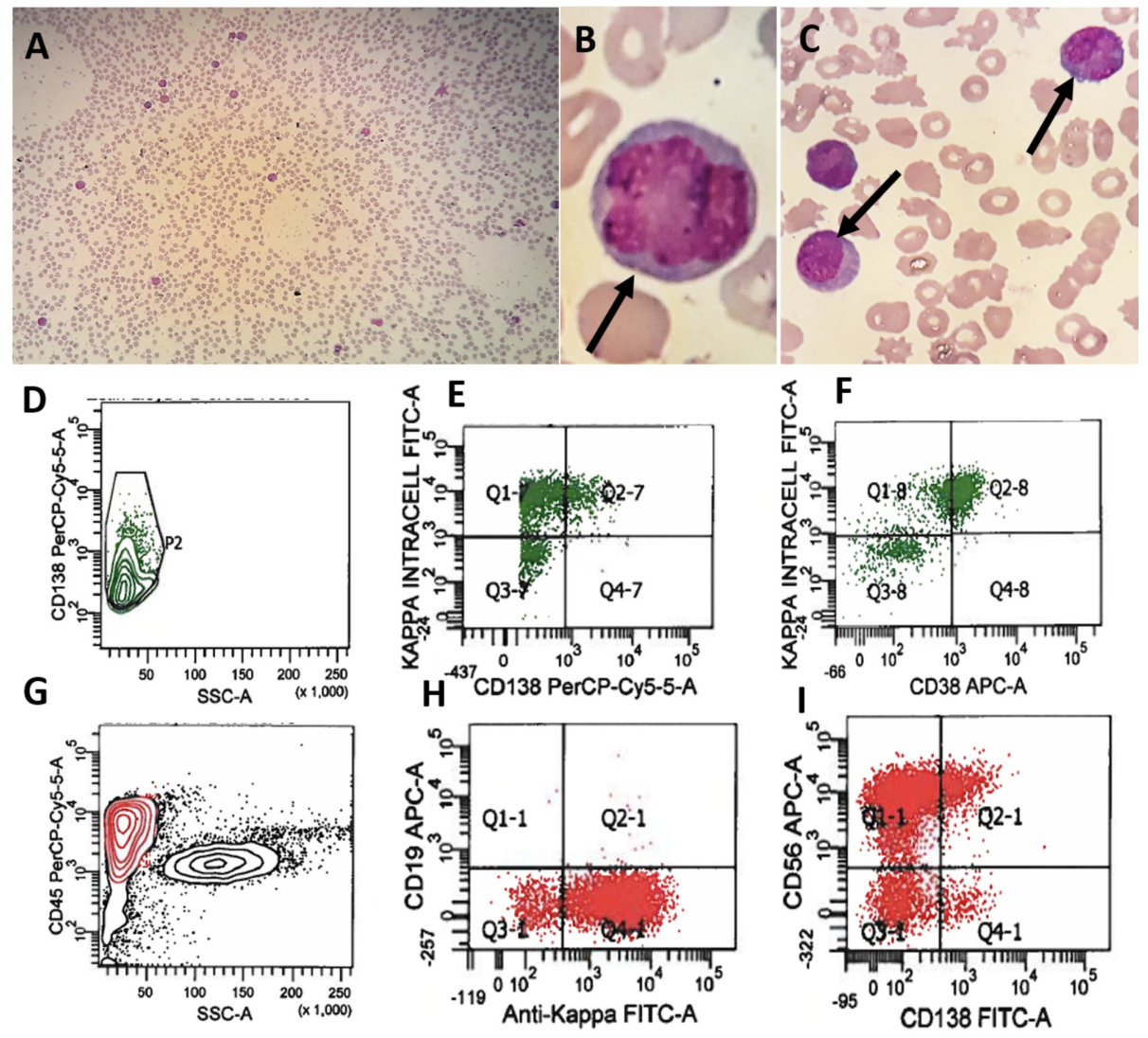

\section{FIGURE 4: Plasma cell leukemia in recurrent multiple myeloma.}

(A-C) Peripheral smear analysis demonstrated leukocytosis (A) and abnormal plasma cells (B, C). (D) Flow cytometry studies showed $22.4 \%$ CD138 dim-positive cells with kappa restriction (E) and dim-positive expression of CD38 (F). (G) The zebra plot showed abnormal cell population with kappa restriction (H) with expression of CD56 and CD138 (dim) markers (I).

The peripheral smear examined one week after completion of two cycles of brentuximab vedotin, etoposide, cytoxan and dexamethasone showed leukocytopenia with near complete elimination of plasma cells from the peripheral smear (Figure 5A). Flow cytometry did not detect a residual CD138-positive population or kappa expressing population (Figures $5 B-5 D$ ). The patient was continued on three cycles of brentuximab vedotin with progressive response with a gradual decrease in serum free light chain (FLC). However, he eventually developed lethargy, left leg weakness and imbalance. The MRI showed multiple metastatic lesions in the brain. He underwent lumbar puncture with cerebrospinal fluid showing atypical cells, but flow cytometry revealed the clonal CD38, CD56, CD45 and intracellular kappa-positive population with aberrant expression of clonal CD4 cells comprising $40 \%$ of total events. These findings confirmed the metastatic involvement of patients with MM. 


\section{Cureus}
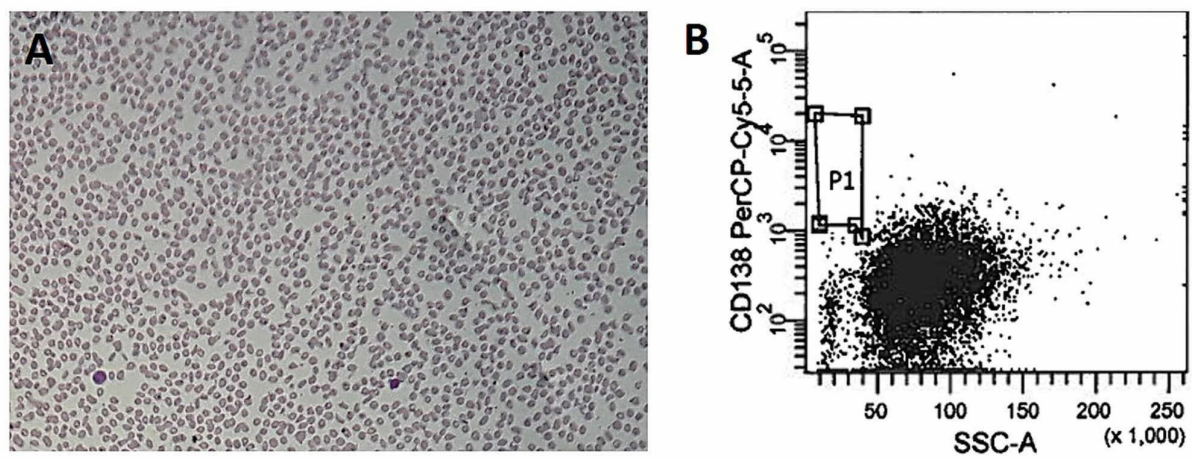

C

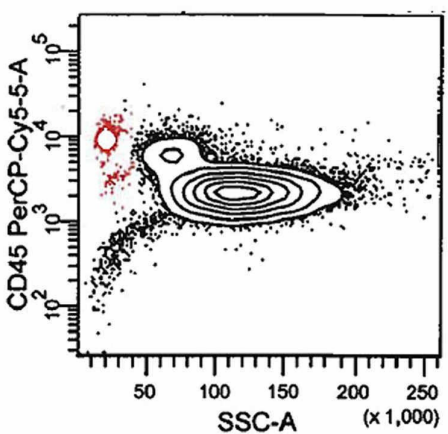

D

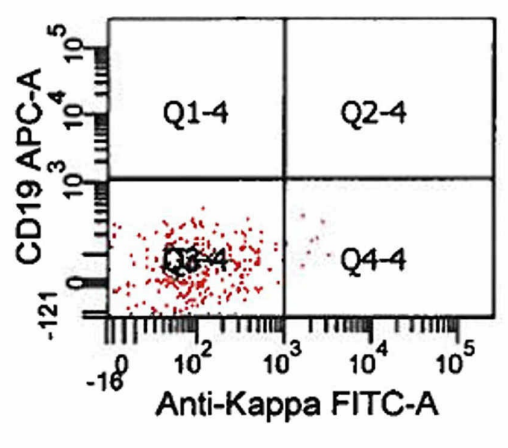

FIGURE 5: Post therapy effect on plasma cell leukemia.

(A) Peripheral smear analysis demonstrated leukocytopenia. (B) Flow cytometry analysis did not show residual CD138-positive cells. (C) The zebra plot did not show residual kappa-positive cells (D).

\section{Discussion}

WHO defines MM at least one or more myeloma-defining events (MDEs) in addition to evidence of either $10 \%$ or more clonal plasma cells on bone marrow examination or a biopsy-proven plasmacytoma. MDE consists of established parameters for CRAB features (hypercalcemia, renal failure, anemia or lytic bone lesions) and other biomarkers of malignancy: clonal bone marrow plasma cells of $60 \%$ or higher, serum FLC ratio of 100 or higher (provided involved FLC level is $\geqslant 100 \mathrm{mg} / \mathrm{L}$ ) and more than one focal lesion on MRI $[2,8]$.

MGUS is considered as a premalignant condition for MM which devoid of clinical criteria associated with MM. MGUS patients usually have $1 \%$ average annual risk of progression to malignant MM. Our patient demonstrated symptom of MM within two years after being diagnosed with MGUS.

PCL is a rare disease and accounts for $1 \%-2 \%$ of all hematological malignancies with a median age of 50-60 years with an approximately equal proportion of male and female patients. Although PCL is defined by the presence of $>2 \times 10^{9}$ /liter of CPCs in peripheral blood or by a relative plasmacytosis $>20 \%$ of blood leukocytes, recent studies have advocated the use of a lower threshold of CPCs to define PCL [3-5]. Despite undergoing cycles of combination of bortezomib, lenalidomide, daratumumab, pomalidomide and dexamethasone for MM, our patient failed to achieve remission. Unfortunately, while on treatment regimen, he developed plasmacytoma of kidney (Figure 3). Furthermore, CD30-positive cells were detected in the plasmacytoma via flow cytometry, and hence brentuximab vedotin was added to the regimen. At our institute, during routine peripheral smear review, $45 \%-55 \%$ of abnormal cells with multilobated morphology were seen (Figures $4 \mathrm{~A}-4 \mathrm{C}$ ). The flow cytometry of peripheral blood confirmed presence of $22.4 \%$ CD138 dimpositive and kappa-restricted plasma cells (Figure $4 D-4 F$ ). These findings were consistent with a diagnosis of sPCL. In our patient, CD38 expression was also dim due to treatment with daratumumab, a anti-CD38 antibody (Figure $4 F$ ) [9]. Interestingly, flow cytometry of the peripheral blood also demonstrated dim CD138 expression (Figure 4D). CD138 molecule is known to disintegrate and disappear quickly in the sample, and hence flow cytometry analysis for CD138 is usually underestimated. Furthermore, expression can be lost during the processing of the sample for flow cytometry; hence, the dim CD138 expression may not represent the true biological expression of CD138 in our patient as well [10]. In addition to the cytoplasmic kappa, almost 70\% of these abnormal cells also expressed surface kappa of which some were positive for CD56 and CD138 markers (Figure 4G-4I). Usually, plasma cells in PCL and MM patients express cytoplasmic light chain clone but plasma cells have also been shown to express surface as well as cytoplasmic light chain clone in other published reports $[10,11]$. In addition, multilobated form of plasma cells seen in our patient peripheral smear has also been reported previously [12]. These findings highlight that SPCL may demonstrate abnormal 
morphology instead of typical plasma cells and require careful assessment of peripheral smear during the management of MM. In addition, the rapid progression from MM to plasmacytoma and then SPCL suggest the aggressive form of MM and require multidisciplinary management and treatment regimen keeping in the mind the phenotype of the abnormal lesion cells.

sPCL usually developed in association with translocations of immunoglobulin heavy chain (IgH) translocation with other chromosomal partners (4p16, 6p21, 11q13, 16q23 and 20q11) with or without associated mutation/deletion in the chromosomes and other genes such as NRAS, KRAS, p53, MYC, NF-kB, etc $[6]$. Especially, higher incidence of $\mathrm{t}(11 ; 14)(\mathrm{q} 13 ; \mathrm{q} 32)$ and other chromosomal abnormalities, such as del (17p13), del (1p21), ampl (1p21), t $(14 ; 12)$ and t(4:14), have also been reported [13].

Although transformation of MM to SPCL is highly unpredictable, failure to respond to initial as well as elevated LDH, low serum albumin, elevated beta 2 microglobulin, hypercalcemia, advance age and increase plasma cells at the time of diagnosis of MM are some of the risk factors for progression to SPCL [6]. PCL with $\mathrm{t}(11 ; 14)$ has highly unpredictable clinical course [7]. Studies indicate that a reduction in CPCs by $50 \%$ within 10 days of treatment and to have no CPCs within four weeks of treatment are associated with relatively longer survival [6,14]. Furthermore, for such patients, addition of bcl-2 inhibitor, venetoclax has recently shown promise [15]. Our patient had a complete workup at the time of diagnosis of initial MM, which demonstrated high $\mathrm{LDH}$, abnormal serum protein analysis and $0.9 \%$ of abnormal plasma cells with only $\mathrm{t}(11$; 14) abnormalities upon bone marrow biopsy (Figure $1 E$ ). The addition of venetoclax to the treatment regimen of our patient could have been another strategy to delay the aggressive disease course. As compared to MM, PCL patients usually have decreased expression of the adhesion molecules NCAM (neural adhesion molecule/CD56) and LFA-1 (leukocyte function-associated antigen-1), and thus have extramedullary accumulation and progression of PCL. Hence, CD56 expression on tumor cells is used as a prognostic factor marker even in patients who have undergone treatment with bortezomib $[6,16]$.

Usually autologous and allogenic stem cell therapy has been associated with longer progression-free survival (PFS) and OS in MM patients but complete remission has been reported in PCL especially of primary form with stem cell therapy although OS was inferior to that of patients with MM [17]. Especially in older patients with PCL, the prognosis of PCL with conventional chemotherapy without novel agents is poor; hence, treatment with novel immunomodulatory drugs and proteasome inhibitors is recommended. Bortezomid rapidly reduces the tumor load, reverses PCL associated complications such as renal failure and hypercalcemia, and has a $69 \%$ of overall response rate [18]. Similarly, lenalidomide and pomalidomide with dexamethasone and bortezomid have been used in relapse refractory cases of MM with an overall response rate of 59\% with a mean duration of response of 13.6 months [18]. In advanced age patients with or without comorbidities, induction, consolidation and maintenance with bortezomib-based regimen have improved the response rate and OS in SPCL and MM patients [18].

Although proteasome inhibitors, immunomodulatory agents and monoclonal antibodies have been able to improve the clinical outcome in MM, clinical remission could still be a challenge with these agents. Our patient failed to achieve remission and was treated with proteasome inhibitors and immunomodulatory agents. Several molecular targets, including surface molecules, adhesion molecules and transmembrane receptors, have been identified for MM. Among these targets, anti-CD38 (isatuximab and daratumab), antiCD56 (lorvotuzumab mertansine), anti-CD138 (indatuximab) alone or in combination with other immunomodulatory agents and program cell death (PD-1) antibody (nivolumab, pembrolizumab and pidilizumab) in clinical trials showed OS ranging from $39 \%$ to $78 \%$ with higher response to combination regimen as compared to monotherapy with monoclonal antibody [19,20]. In addition, chimeric antigen receptor T cell (CART) therapy targeting B-cell maturation antigen (BCMA), CD19, KLC and CD138 have shown promising results in phase I clinical trials, especially forrelapse/recurrent MM patients. BCMA-based CART has also shown to eradicate plasmacytoma in some patients [20]. LCAR-B38M CART containing a CAR construct with scFv targeting two BCMA epitopes, $\mathrm{VHH} 1$ and $\mathrm{VHH} 2$, demonstrated an $88 \%$ of overall response rate with minimal adverse events including grade 1 cytokine release syndrome [20].

Our patient had a rapid progression of MM to sPCL and then spread of MM to CNS. To our knowledge, such unique case scenario with unpredictable clinical course of MM has never been reported previously. Being 79-year-old with comorbid conditions, the patient underwent treatment regimen consisting of proteasome inhibitors and immunomodulatory agents but was not able to enroll into clinical trials for treatment with anti-MM antibodies or CART therapy or even bone marrow transplantation. Our case report highlights the clinical scenario where management of MM due to its aggressive clinical course is indeed a great challenge.

\section{Conclusions}

Despite being a rare entity, diagnosis and treatment of SPCL requires careful consideration in cases presenting with recurrent MM, especially in the patients with advanced age and comorbidities. The treatment regimen comprised of combination of novel immunomodulatory agents and monoclonal antibodies has shown improved OS rate. The results from the ongoing clinical trials with CART targeting BCMA, CD19, KLC and CD138 are promising for therapy of refractory/relapsed MM, especially in patients with aggressive clinical course. Our case report underscores the challenge with respect to treatment and management for MM especially when it progresses to SPCL within a short period of time. 


\section{Additional Information \\ Disclosures}

Human subjects: Consent was obtained by all participants in this study. Conflicts of interest: In compliance with the ICMJE uniform disclosure form, all authors declare the following: Payment/services info: All authors have declared that no financial support was received from any organization for the submitted work. Financial relationships: All authors have declared that they have no financial relationships at present or within the previous three years with any organizations that might have an interest in the submitted work. Other relationships: All authors have declared that there are no other relationships or activities that could appear to have influenced the submitted work.

\section{References}

1. Kyle RA, Maldonado JE, Bayrd ED: Plasma cell leukemia: report on 17 cases . Arch Intern Med. 1974, 133:813818.

2. Swerdlow SH, Campo E, Harris NL, et al.: World Health Organization Classification of Tumours of Haematopoietic and Lymphoid Tissues, 4th Edition. IARC Press, Lyon; 2017.

3. An G, Qin X, Acharya C, et al.: Multiple myeloma patients with low proportion of circulating plasma cells had similar survival with primary plasma cell leukemia patients. Ann Hematol. 2015, 94:257-264. 10.1007/s00277-014-2211-0

4. Granell M, Calvo X, Garcia-Guiñón A, et al.: Prognostic impact of circulating plasma cells in patients with multiple myeloma: implications for plasma cell leukemia definition. Haematologica. 2017, 102:1099-1104. 10.3324/haematol.2016.158303

5. Ravi P, Kumar SK, Roeker L, et al.: Revised diagnostic criteria for plasma cell leukemia: results of a Mayo Clinic study with comparison of outcomes to multiple myeloma. Blood Cancer J. 2018, 8:116. 10.1038/s41408-018-0140-1

6. van de Donk NW, Lokhorst HM, Anderson KC, Richardson PG: How I treat plasma cell leukemia. Blood. 2012, 120:2376-2389. 10.1182/blood-2012-05-408682

7. An G, Xu Y, Shi L, et al.: $\mathrm{t}(11 ; 14)$ multiple myeloma: a subtype associated with distinct immunological features, immunophenotypic characteristics but divergent outcome. Leuk Res. 2013, 37:1251-1257. 10.1016/j.leukres.2013.06.020

8. Rajkumar SV: Multiple myeloma: every year a new standard? . Hematol Oncol. 2019, 37:62-65. 10.1002/hon.2586

9. Sanchez L, Wang Y, Siegel DS, Wang ML: Daratumumab: a first-in-class CD38 monoclonal antibody for the treatment of multiple myeloma. J Hematol Oncol. 2016, 9:51. 10.1186/s13045-016-0283-0

10. Kumar S, Kimlinger T, Morice W: Immunophenotyping in multiple myeloma and related plasma cell disorders. Best Pract Res Clin Haematol. 2010, 23:433-451. 10.1016/j.beha.2010.09.002

11. Ocqueteau M, San Miguel JF, Gonzalez M, Almeida J, Orfao A: Do myelomatous plasma cells really express surface immunoglobulins?. Haematologica. 1996, 81:460-463.

12. Fujimi A, Nagamachi Y, Yamauchi N, Kanisawa Y: Morphological transformation of myeloma cells into multilobated plasma cell nuclei within 7 days in a case of secondary plasma cell leukemia that finally transformed as anaplastic myeloma. Case Rep Hematol. 2017, 2017:5758368. 10.1155/2017/5758368

13. Gundesen MT, Lund T, Moeller HEH, Abildgaard N: Plasma cell leukemia: definition, presentation, and treatment. Curr Oncol Rep. 2019, 21:8. 10.1007/s11912-019-0754-X

14. de Larrea CF, Kyle RA, Durie BG, et al.: Plasma cell leukemia: consensus statement on diagnostic requirements, response criteria and treatment recommendations by the International Myeloma Working Group. Leukemia. 2013, 27:780-791. 10.1038/leu.2012.336

15. Durie BG, Goldschmidt H, Mateos MV, et al.: Outcomes of patients with $\mathrm{t}(11 ; 14)$ multiple myeloma: an International Myeloma Working Group (IMWG) multicenter study. J Clin Oncol. 2019, 37:8015-8015.

16. Skerget M, Skopec B, Zadnik V, et al.: CD56 expression is an important prognostic factor in multiple myeloma even with bortezomib induction. Acta Haematol. 2018, 139:228-234. 10.1159/000489483

17. Drake MB, Iacobelli S, van Biezen A, et al.: Primary plasma cell leukemia and autologous stem cel transplantation. Haematologica. 2010, 95:804-809. 10.3324/haematol.2009.013334

18. Katodritou E, Terpos E, Kelaidi C, et al.: Treatment with bortezomib-based regimens improves overall response and predicts for survival in patients with primary or secondary plasma cell leukemia: analysis of the Greek myeloma study group. Am J Hematol. 2014, 89:145-150. 10.1002/ajh.23600

19. Hajek R: Strategies for the treatment of multiple myeloma in 2013: moving toward the cure . Multiple Myeloma. IntechOpen, London; 2013. 1-12.

20. Sohail A, Mushtaq A, Iftikhar A, et al.: Emerging immune targets for the treatment of multiple myeloma . Immunotherapy. 2018, 10:265-282. 10.2217/imt-2017-0136 